\title{
Método Pilates na funcionalidade do membro superior de mulheres mastectomizadas
}

Pilates method on the functionality of the superior member of mastectomized women

Método de Pilates sobre la funcionalidad de las extremidades superiores en mujeres mastectomizadas

Kalilia Cristina das Merces Ferreira ${ }^{1 *}$, Ana Paula Nascimento dos Santos ${ }^{1}$, Michelle Castro da Silva Holanda $^{2}$, Patrick Roberto Gomes Abdoral ${ }^{3}$, Larissa Siqueira Rodrigues ${ }^{1}$, Cybelle Silva do Couto Coelho $^{3}$, Iranete Corpes Oliveira França ${ }^{1}$, Mayra Herminia Simões Hamad Farias do Couto ${ }^{1}$, Cláudia Jeane Claudino de Pontes Miranda ${ }^{1}$, Susanne Cristine Brito e Silva ${ }^{1}$.

\section{RESUMO}

Objetivo: Avaliar a eficácia do Método Pilates na funcionalidade de mulheres submetidas à Mastectomia Radical. Métodos: Trata-se de um ensaio clínico com participantes na faixa etária entre 30 e 61 anos, que realizaram um programa de reabilitação fisioterapêutica através do Método Pilates em um período de 3 meses. Antes e após o protocolo de tratamento, avaliou-se a amplitude de movimento através de Goniometria, teste de força muscular manual e funcionalidade por meio da Medida de Independência Funcional (MIF). Resultados: 22 participantes foram avaliadas, destas 11 foram excluídas: 3 pacientes por não preencherem os critérios de inclusão; 6 por excesso de faltas e 2 desistências. As 11 pacientes que concluíram o programa de reabilitação, apresentaram melhora significativa na amplitude dos movimentos do ombro (flexão, extensão, abdução e adução) ( $p<0.05)$, flexo-extensão do cotovelo e inclinação lateral da coluna cervical $(p<0.05)$, além de incremento na independência funcional $(p<0.004)$ e força muscular para os principais grupos musculares: flexores, extensores, abdutores e rotadores internos de ombro $(p<0.0001)$. Conclusão: O Método do Pilates mostrou-se eficaz nesta amostra na melhora da funcionalidade de mulheres submetidas à Mastectomia Radical, promovendo ganho na amplitude de movimento e da força.

Palavras-chave: Neoplasias da mama, Terapia por exercício, Mastectomia radical, Método Pilates.

\begin{abstract}
Objective: To evaluate the effectiveness of the Pilates Method on the functionality of women undergoing Radical Mastectomy. Methods: This is a clinical trial with participants aged between 30 and 61 years, who underwent a physical therapy rehabilitation program using the Pilates Method, two to three times a week, lasting 60 minutes, totaling 24 sessions in one period 3 months. Before and after the treatment protocol, range of motion was assessed using Goniometry, manual muscle strength test and functionality using the Functional Independence Measure. Results: 22 participants were evaluated, of which 11 were excluded: 3 patients for not meeting the inclusion criteria; 6 due to excessive absences and 2 dropouts. The 11 patients who completed the rehabilitation program showed a significant improvement in the range of shoulder movements (flexion, extension, abduction and adduction) $(p<0.05)$, elbow flexion-extension and lateral inclination of the cervical
\end{abstract}

${ }^{1}$ Universidade da Amazônia (UNAMA), Belém - PA. *E-mail: kaliliamferreira@gmail,com

2 Universidade do Estado do Pará (UEPA), Belém - PA.

${ }^{3}$ Faculdade Cosmopolita, Belém - PA.

SUBMETIDO EM: 11/2021

ACEITO EM: 12/2021

PUBLICADO EM: 12/2021 
spine $(p<0.05)$, in addition to an increase in functional independence $(p<0.004)$ and muscle strength for the main muscle groups: flexors, extensors, abductors and internal shoulder rotators $(p<0.0001)$. Conclusion: The Pilates Method proved to be effective in this sample in improving the functionality of women undergoing Radical Mastectomy, promoting gains in range of motion and strength.

Keywords: Breast neoplasms, Exercise therapy, Mastectomia radical, Pilates method.

\section{RESUMEN}

Objetivo: Evaluar la efectividad del Método Pilates sobre la funcionalidad de las mujeres sometidas a Mastectomía Radical. Métodos: Este es un ensayo clínico con participantes de edades comprendidas entre 30 y 61 años, que se sometieron a un programa de fisioterapia utilizando el Pilates en un período 3 meses Antes y después del protocolo de tratamiento, el rango de movimiento se evaluó mediante Goniometría, prueba de fuerza muscular manual y funcionalidad utilizando la Medida de Independencia Funcional. Resultados: 22 participantes fueron evaluados, de estos 11 fueron excluidos: 3 pacientes por no cumplir con los criterios de inclusión; 6 debido a ausencias excesivas y 2 abandonos. Los 11 pacientes que completaron el programa de rehabilitación mostraron una mejora significativa en el rango de movimientos del hombro ( $p$ $<0.05)$, flexión-extensión del codo e inclinación lateral de la columna cervical $(p<0.05)$, además de un aumento en la independencia funcional $(p<0.004)$ y la fuerza muscular para los principales grupos musculares: flexores, extensores, abductores y rotadores internos del hombro $(p<0.0001)$. Conclusión: El Método Pilates demostró ser efectivo en esta muestra para mejorar la funcionalidad de las mujeres sometidas a Mastectomía Radical, promoviendo ganancias en el rango de movimiento y fuerza.

Palabras clave: Neoplasias de la mama, Terapia por ejercicio, Mastectomía radical, Método de Pilates.

\section{INTRODUÇÃO}

O câncer de mama é a doença resultante de multiplicação desordenada de células região mamária, capaz de atingir ambos os sexos, mas com maior incidência em mulheres acima de 40 anos de idade. Apresenta alta taxa de mortalidade, com incidência ponderada de estimada em 59.700 casos novos no ano de 2019, sendo que 29,5\% referente ao câncer de mama feminino no Brasil (GUERRA MR, et al., 2015; INSTITUTO NACIONAL DE CÂNCER (INCA), 2019).

Existem vários fatores de risco que podem influenciar no aparecimento ou evolução do câncer de mama, sendo o envelhecimento, um dos mais agravantes na mulher, em função das alterações hormonais que também funcionam como fatores de risco, bem como a menarca precoce, a nuliparidade, a primeira gestação após os 30 anos de idade, a diminuição dos níveis de estrogênio e testosterona, provocando a menopausa e, posteriormente, o climatério. Os fatores ambientais também favorecem condições para disseminação da patologia, entre eles a exposição solar, estilo de vida, o consumo de álcool e ingestão exagerada de gorduras saturadas poluição, a contínua exposição às radiações ionizantes. Os principais sinais e sintomas dessa doença são o aparecimento de nódulos mamários axilares e no pescoço, eritema na pele e líquido anormal produzido pela mama (ALVES ERP, et al., 2015; MINISTÉRIO DA SAÚDE, 2017).

Atualmente, um dos principais tratamentos é a mastectomia, procedimento caracterizado como uma "desconfiguração" do corpo da mulher, que causa sequela traumática e influenciam na recuperação, podendo haver o aparecimento de fibrose cicatricial, dor, alterações de sensibilidade e na mobilidade do membro superior, sendo a realização da fisioterapia pós-mastectomia um fator importante na recuperação (ALMEIDA TG, et al., 2015).

No âmbito da fisioterapia, o Pilates tem se mostrado como um recurso viável no tratamento de diversas disfunções do corpo humano como: lombalgias, astenias, alterações posturais, etc. Diferenciando-se através de seus fundamentos para realização dos exercícios, e se mostrando eficaz na melhora da consciência postural, Amplitude De Movimento (ADM), fortalecimento muscular e na regulação do ciclo respiratório. $O$ 
método pode ser indicado aos chamados "grupos especiais", como as gestantes e os idosos, pois os exercícios são adaptáveis de acordo com a situação clínica do paciente, levando em consideração as características e habilidades que ele desenvolve durante a prática do Método (JESUS LT, et al., 2015).

A fisioterapia possui técnicas amplamente utilizadas na prática clínica, que se mostraram eficientes na melhora da funcionalidade de pacientes submetidas mastectomia radical, e muito importante ressaltar a necessidade da abordagem fisioterapêutica no pré e pós-operatório imediato e tardio (CASASSOLA GM, et al., 2020).

As condutas da fisioterapia são essenciais no tratamento para as complicações geradas no pósmastectomia radical, o acompanhamento dessas pacientes a curto e longo prazo torna o processo de reabilitação mais eficiente. Técnicas como drenagem linfática manual, enfaixamento compressivo, cuidados com a pele podem estar relacionados aos exercícios cinesioterápico como o método pilates resultando em uma eficácia mais significativa na redução das complicações (GUGELMIN MRG, 2018).

Neste contexto, o Método Pilates tem efeitos significativos quando aplicados em mulheres mastectomizadas, melhorando os aspectos físicos e também psicossociais, tornando-o uma intervenção segura. Assim, o objetivo deste estudo foi avaliar a eficácia do Método Pilates na funcionalidade, ADM e força muscular do membro superior de mulheres submetidas à Mastectomia Radical.

\section{MÉTODOS}

Trata-se de um ensaio clínico, quantitativo, longitudinal de um grupo amostral, por conveniência, aprovado pelo Comitê de Ética da Universidade da Amazônia, sob número 2.603.786.

Foram selecionadas pessoas do sexo feminino, faixa etária entre 30 e 65 anos, com diagnóstico de câncer de mama, que realizaram Mastectomia Radical, e que estivessem a partir de 30 dias de pós-cirúrgico. Foram excluídas as mulheres que apresentassem alteração na cicatrização, quadro álgico acentuado (8 a 10, de acordo com a escala visual analógica), que realizassem outro tipo de tratamento, ou aquelas que faltassem por mais de três vezes aos atendimentos, desistentes, com recidivas de doenças locais e que não tolerassem o protocolo de tratamento.

Para a coleta de dados, foi utilizada uma ficha de avaliação fisioterapêutica construída pelas pesquisadoras com os itens: anamnese e exame físico composto por Goniometria (exame manual da coluna cervical, ombro e cotovelo em movimento ativo, com goniômetro Carcißi)).

Também foi realizado o Teste de força muscular manual, para flexores de ombro (deltoide anterior, coracobraquial e bíceps braquial), extensores de ombro (deltoide posterior, tríceps braquial, grande dorsal, romboide maior e menor), abdutores (deltoide médio e supra-espinhal), adutores (peitoral maior e grande dorsal), rotadores externos (redondo menor, infra espinhal, além dos músculos serrátil anterior e abdutor da escápula) e rotadores interno (redondo maior, subescapular e grande dorsal) de ombro; músculos flexoextensores de cotovelo (bíceps braquial, braquial, braquiorradial, tríceps braquial e ancôneo), e extensores (Trapézio, esplênio da cabeça, esplênio do pescoço e semi-espinhal) de cervical. A avaliação da dor foi realizada no início e no final de cada sessão através da aplicação da Escala Visual Analógica (EVA).

Após a avaliação, aplicou-se a Medida de Independência Funcional (MIF), instrumento utilizado para avaliar a incapacidade funcional de diferentes causas e patologias, dividida em dois domínios, cognitivo e motor, contendo 18 itens, agrupado em 6 dimensões: autocuidado, locomoção, transferência, comunicação, controle dos esfíncteres e cognição social. Cada item pode receber uma pontuação de 1 a 7 , respectivamente, dependência ou independência completa. O escore é dado pela soma de valores que varia de 18 a 126 , onde 18: dependência completa; 19 a 60: dependência modificada; 61 a 103: independência modificada e 104 a 126: independência completa/modificada (ASSIS CS, et al., 2015).

O protocolo de reabilitação foi realizado duas a três vezes na semana, com duração de 60 minutos, totalizando 24 sessões. Durante os atendimentos, foram realizadas orientações e/ou correções quanto aos 
exercícios e aos princípios do Método. Os equipamentos utilizados foram: Cadillac, Reformer e a Chair adicionados aos acessórios magic circle e meia-lua, que foram utilizados nas variações de cada exercício, para facilitar ou dificultar sua execução. Os exercícios selecionados foram classificados em fases I e II de acordo com cada equipamento.

Ao iniciar cada atendimento, foi realizado um grupo de exercícios chamado "Pré-Pilates", com duração de 5 minutos, auxiliando a lubrificar as articulações, com os movimentos de rotação, flexão lateral, extensão e flexão cervical; correção da postura dos ombros e pescoço, abdução dos ombros, flexão e extensão do tornozelo em posição ortostática.

O protocolo iniciou na fase I, em que a paciente deveria conseguir realizar os exercícios básicos, tendo aprendido o padrão respiratório proposto pelo Método, assim como realizar a ativação do Powerhouse, ensinado no início do protocolo, e iniciar os movimentos de aumento de força muscular para os membros superiores e inferiores. Em seguida, foi iniciada a fase II, em que a paciente deveria realizar a ativação do Powerhouse, incorporando respiração, durante os movimentos, que deveriam ser fluidos e coordenados, do centro para as extremidades.

Nos 5 minutos finais, era realizado exercício de alongamento muscular na cadeia anterior, posterior e lateral do corpo em posição de repouso, a fim de realizar o relaxamento.

Os dados coletados foram tabulados, interpretados, processados e analisados por meio da estatística descritiva e inferencial. Para a análise, foram utilizados Microsoft Excele Statistic Package for Social Sciences (SPSS), versão 24.0, todos em ambiente Windows 7.

Para análise dos escores médios, segundo funcionalidade e ADM, foi utilizado o teste t de Student para comparação de médias. Este teste busca apontar quais médias, de diferentes grupos e variáveis, a partir do escore obtido nas escalas de avaliação, apresentam diferenças significativas, ao nível de significância de $95 \%$ (AYRES M, et al., 2015).

\section{RESULTADOS}

Foram avaliadas 22 mulheres, das quais 11 foram excluídas: 3 pacientes por não preencherem os critérios de inclusão; 6 por excesso de faltas e 2 desistências, restando 11 pacientes que concluíram todo o protocolo. Observou-se, que $45,55 \%$ apresentavam o membro superior direito afetado, $36,36 \%$ o esquerdo e $18,18 \%$ os dois membros afetados.

Pôde-se verificar que as mulheres submetidas à Mastectomia Radical possuíam, um valor de média $\mu=$ 44.73 , com desvio padrão, $\pm \mathrm{DP}=7.38$. O grupo possuía idade mínima de 34 anos e a máxima de 61 anos. Segundo a mediana, $50 \%$ das mulheres têm mais de 41 anos.

No que tange a avaliação da amplitude de movimento, a Tabela 1 mostra que as médias obtidas para quase todos os membros avaliados se mostrou significativamente maior $(p<0.05)$ quando comparados com a avaliação antes e após a aplicação do tratamento com o método pilates.

As diferenças estatisticamente significativas encontradas foram: na flexão de ombro, entre as mulheres afetadas bilateralmente, na extensão de ombro, entre as mulheres afetadas bilateralmente, e entre aquelas afetadas no lado direito, também na abdução de ombro, em mulheres afetadas bilateralmente, e nas mulheres afetadas no lado direito e entre aquelas afetadas no lado esquerdo $(p<0.05)$.

$\mathrm{Na}$ adução de ombro, apenas as mulheres afetadas no lado esquerdo apresentaram diferença significativa na avaliação goniométrica. No movimento de flexo-extensão de cotovelo, as mulheres afetadas bilateralmente e as afetadas no lado esquerdo apresentaram diferença significativa $(p<0.05)$. Na inclinação lateral de cervical, as mulheres afetadas bilateralmente e as mulheres afetadas no lado esquerdo, apresentaram diferença significativa na avaliação goniométrica, conforme Tabela 1. 
Tabela 1 - Comparação de médias das variáveis de ADM do membro superior e coluna Cervical de mulheres submetidas à Mastectomia Radical, segundo o lado afetado, considerando a avaliação inicial e a final, em uma clínica particular no estado do Pará, 2019, n= 11 participantes.

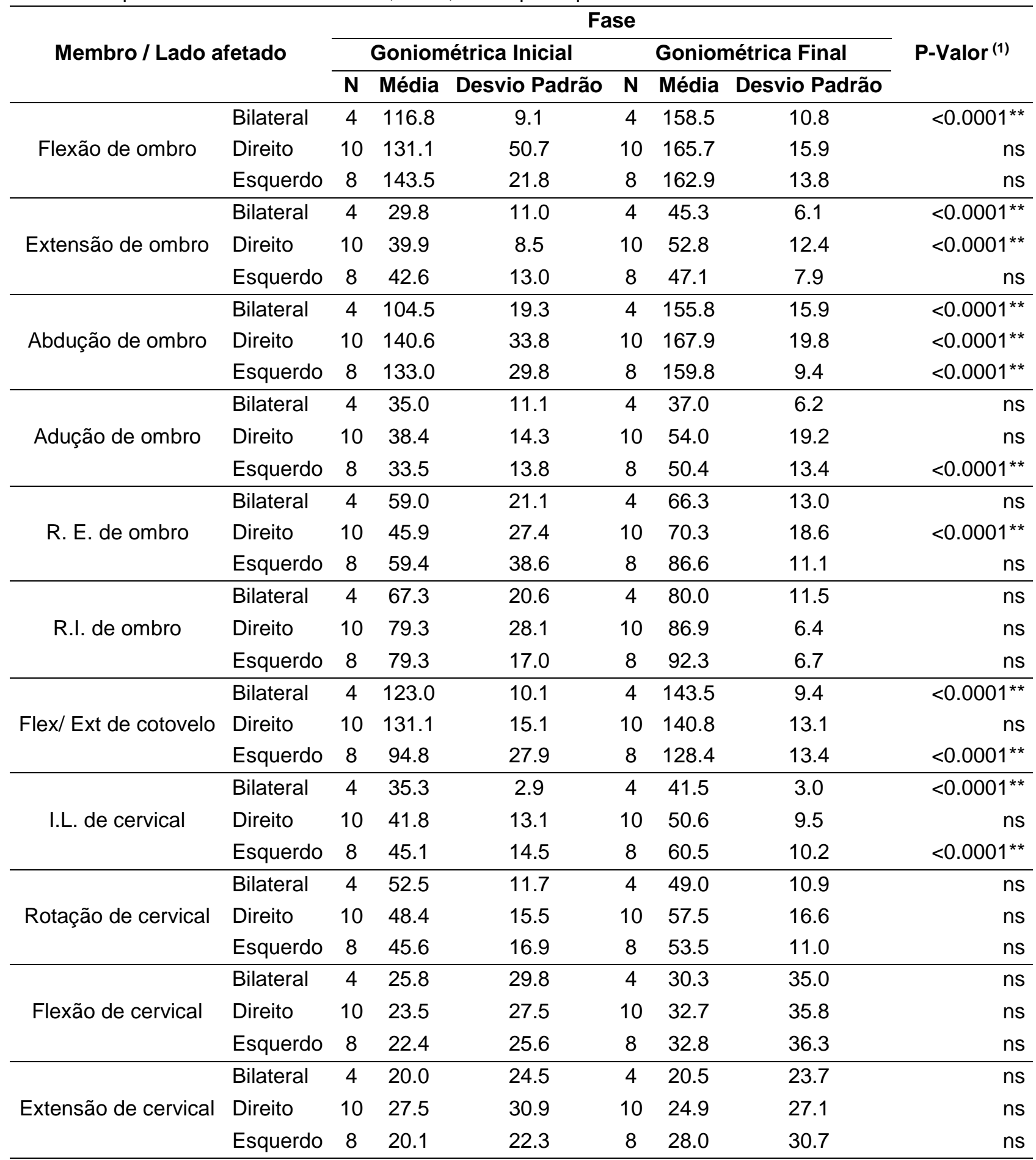

Nota: (1) Teste t de Student para comparação de médias ( $p$-valor<0.05). ${ }^{* *}$ Valores Altamente significativos; NS: Valores Não Significativos. Legenda: R.E: Rotação Externa; R.I: Rotação interna; I.L: Inclinação Lateral; $\mathrm{N}$ : número de participantes. Fonte: Ferreira KCM, et al., 2021.

Em relação aos achados quanto à força muscular, houve diferenças estatística significativas para os principais grupos musculares: flexores, extensores, abdutores e rotadores internos de ombro $(p<0.0001)$. Nas mulheres, cujo lado afetado era o direito, houve melhora na adução do ombro, e naquelas cujo membro afetado era o esquerdo, obteve-se aumento de força no movimento de rotação externa de ombro (Tabela 2). 
Tabela 2 - Comparação de médias da força muscular de mulheres submetidas à Mastectomia Radical, segundo o lado afetado, considerando a avaliação inicial e a final, em uma clínica particular no estado do Pará, 2019, $n=11$ participantes.

\begin{tabular}{|c|c|c|c|c|c|c|c|c|}
\hline \multirow{2}{*}{\multicolumn{2}{|c|}{ Membro / Lado afetado }} & \multicolumn{6}{|c|}{ Força Muscular } & \multirow[t]{3}{*}{ P-Valor (1) } \\
\hline & & \multicolumn{3}{|c|}{ Inicial } & \multicolumn{3}{|c|}{ Final } & \\
\hline & & $\mathbf{N}$ & Média & Desvio Padrão & $\mathbf{N}$ & Média & Desvio Padrão & \\
\hline \multirow{3}{*}{$\begin{array}{l}\text { Flexão de } \\
\text { ombro }\end{array}$} & Bilateral & 2 & 3.0 & 0.0 & 2 & 4.5 & 0.57 & $<0.0001^{\star \star}$ \\
\hline & Direito & 5 & 3.6 & 0.54 & 5 & 4.6 & 0.54 & $<0.0001^{* *}$ \\
\hline & Esquerdo & 4 & 3.25 & 1.25 & 4 & 5.0 & 0.0 & $<0.0001^{* *}$ \\
\hline \multirow{3}{*}{$\begin{array}{l}\text { Extensão } \\
\text { de ombro }\end{array}$} & Bilateral & 2 & 2.75 & 0.5 & 2 & 4.5 & 0.57 & $<0.0001^{* *}$ \\
\hline & Direito & 5 & 3.8 & 0.83 & 5 & 4.6 & 0.54 & $<0.0001^{* *}$ \\
\hline & Esquerdo & 4 & 3.25 & 1.25 & 4 & 4.75 & 0.5 & $<0.0001^{* *}$ \\
\hline \multirow{3}{*}{$\begin{array}{l}\text { Abdução de } \\
\text { ombro }\end{array}$} & Bilateral & 2 & 3.5 & 0.57 & 2 & 4.5 & 0.57 & $<0.0001^{* *}$ \\
\hline & Direito & 5 & 3.8 & 0.83 & 5 & 5.0 & 0.0 & $<0.0001^{* *}$ \\
\hline & Esquerdo & 4 & 3.25 & 1.25 & 4 & 4.75 & 0.5 & $<0.0001^{* *}$ \\
\hline \multirow{3}{*}{$\begin{array}{l}\text { Adução de } \\
\text { ombro }\end{array}$} & Bilateral & 2 & 3.25 & 0.5 & 2 & 4.0 & 1.15 & ns \\
\hline & Direito & 5 & 4.2 & 0.83 & 5 & 5.0 & 0.0 & $<0.0001^{* *}$ \\
\hline & Esquerdo & 4 & 3.75 & 1.25 & 4 & 4.75 & 0.5 & $\mathrm{Ns}$ \\
\hline \multirow{3}{*}{$\begin{array}{l}\text { R. I. de } \\
\text { ombro }\end{array}$} & Bilateral & 2 & 3.5 & 0.57 & 2 & 4.5 & 0.57 & $<0.0001^{* *}$ \\
\hline & Direito & 5 & 3.6 & 0.89 & 5 & 4.6 & 0.54 & $<0.0001^{* *}$ \\
\hline & Esquerdo & 4 & 3.5 & 1.29 & 4 & 4.75 & 0.5 & $<0.0001^{* *}$ \\
\hline \multirow{3}{*}{$\begin{array}{l}\text { R.E. de } \\
\text { ombro }\end{array}$} & Bilateral & 2 & 4.0 & 1.15 & 2 & 4.5 & 0.57 & ns \\
\hline & Direito & 5 & 4.2 & 1.09 & 5 & 5.0 & 0.0 & ns \\
\hline & Esquerdo & 4 & 3.25 & 1.25 & 4 & 4.75 & 0.5 & $<0.0001^{* *}$ \\
\hline Extensão & Bilateral & 2 & 4.0 & 0.7 & 2 & 5.0 & 0.0 & ns \\
\hline Extensão & Direito & 5 & 4.0 & 2.6 & 5 & 5.0 & 2.2 & ns \\
\hline de cervical & Esquerdo & 4 & 4.25 & 0.5 & 4 & 5.0 & 0.0 & ns \\
\hline
\end{tabular}

Nota: (1) Teste t de Student para comparação de médias (p-valor $<0.05) .{ }^{* *}$ Valores Altamente significativos; NS: Valores Não Significativos. Legenda: R.E: Rotação Externa; R.I: Rotação interna; I.L: Inclinação Lateral; N: número de participantes. Fonte: Ferreira KCM, et al., 2021.

O principal objetivo da fisioterapia com o método Pilates nas pacientes submetidas à Mastectomia Radical e a retomada das suas atividades realizadas com independência e funcionalidade, podemos verificar essas informações na Tabela 3 que mostra na fase final as mulheres se mostraram mais independentes funcionalmente $(p<0.05)$.

Tabela 3 - Comparação de médias da Medida de Independência Funcional do membro superior de mulheres submetidas à Mastectomia Radical, segundo a avaliação inicial e a final, em uma clínica particular no estado do Pará, 2019, $\mathrm{n}=11$ participantes.

\begin{tabular}{lcccccccc}
\hline Variável & Média & DP & Mín. & Med. & Máx. & Dif. & IC de 95\% & P-Valor $^{(1)}$ \\
\hline MIF (PI) & 85.91 & 3.65 & 79 & 86 & 91 & -4.64 & $(-7.58 ;-1.69)$ & \multirow{2}{*}{$0.004^{* *}$} \\
MIF (PF) & 90.545 & 2.876 & 85 & 91 & 97 & & & \\
\hline
\end{tabular}

Nota: (1) Teste t de Student para comparação de médias ( $p$-valor $<0.05) .{ }^{*}$ Valores Altamente significativos. Legenda: PI: Pontuação Inicial; PF: Pontuação Final; DP: Desvio Padrão; IC: Intervalo de Coeficiente.

Fonte: Ferreira KCM, et al., 2021.

Através dos resultados obtidos Tabela $3 \mathrm{com}$ as 11 pacientes, podemos verificar que tivemos um importante melhora na funcionalidade de independências, isso repercute uma melhora na qualidade de vida e na autoestima das participantes do estudo. 


\section{DISCUSSÃO}

O presente estudo abordou mulheres pós-mastectomia radical, na faixa etária de 34 a 61 anos, com ambos os membros superiores afetados ou apenas um, que evoluíram com melhora na mobilidade articular dos membros superiores e coluna cervical, aumento de força muscular e na independência funcional, após a aplicação do programa de tratamento proposto com o método Pilates.

No que se refere à faixa etária das participantes desta pesquisa, variou entre 34 a 61 anos de idade, encontrando-se valores inferiores ao que a literatura aponta enquanto maior incidência do câncer de mama, na faixa de 40 a 69 anos, isso porque as mulheres têm maior de exposição do corpo ao estrogênio devido as características reprodutivas, tonando a dependente, o que pode predispor ao câncer de mama (PEREIRA LKNV, et al., 2015).

Em relação à amplitude de movimento avaliado nesse estudo após as 24 sessões de Pilates realizadas demonstraram-se aumento na mobilidade articular pela facilidade encontrada, devido o grupo amostral ser de mulheres jovens, pelo tempo de aplicação do protocolo e a quantidade de vezes realizada por semana, obteve o incremento da angulação dos movimentos de flexão, extensão, abdução, adução e rotação externa de ombro e ainda, flexo-extensão de cotovelo e inclinação lateral de cervical.

Entre as complicações mais frequentes nos estudos sobre mastectomia evidenciaram que a redução de força muscular, a limitação na amplitude de movimento, a presença de dor e a aparecimento de linfedema influenciam negativamente a qualidade de vida das pacientes (FIREMAN KM, et al., 2018).

As condutas da fisioterapia são essenciais no tratamento para as complicações geradas no pósmastectomia radical, o acompanhamento dessas pacientes a curto e longo prazo torna o processo de reabilitação mais eficiente. Técnicas como drenagem linfática manual, enfaixamento compressivo, cuidados com a pele podem estar relacionados aos exercícios cinesioterápico como o método pilates resultando em uma eficácia mais significativa na redução das complicações (GUGELMIN MRG, 2018).

A qualidade de vida tem um conceito amplo, complexo e multifatorial. Como foi possível observar no estudo de Fireman KM, et al. (2018) após o câncer e seu complicado tratamento, as vidas das pacientes foram modificadas severamente, após a realização do ciclo de tratamento com a fisioterapia todas as 29 pacientes relataram impacto positivo do tratamento no desempenho das suas atividades de vida diária e domésticas, sentem-se mais seguras e independentes para retomarem suas rotinas

O aumento na amplitude de movimento encontrado no presente estudo e na literatura pode ser devido à atuação do exercício terapêutico na medida em que este age na mobilização do ombro de forma livre respeitando o limite de dor da paciente (FERNANDES ACS, et al., 2017).

Num estudo sobre o perfil epidemiológico de mulheres mastectomizadas relata sobre as consequências físicas e emocionais desfavoráveis à vida da mulher, tais como: lesões musculares, hemorragias, complicações cicatriciais, alterações na sensibilidade, fibroses, alterações posturais, algias, diminuição ou perda total da amplitude de movimento e da força muscular, comprometimento da capacidade respiratória, perda ou redução da capacidade funcional e linfedema do braço homolateral a cirurgia (LENA PR, et al., 2018). Para Ferreira TN (2017) o Pilates busca a realização dos movimentos de forma eficiente, correta e controlada, capaz de ativar todo o corpo, sem causar a exaustão ou a fadiga, deixando de lado o grande número de repetições e carga elevada, buscando sempre a conexão do corpo de forma global e a mente.

De acordo com Branco ANC, et al. (2017), o método Pilates como ouso de exercícios funcionais e atividades circenses melhora da flexibilidade muscular, sendo capaz de melhorar a flexibilidade muscular de tronco e da cadeia posterior a curto prazo e com isso a força muscular era um fator primordial para esse ganho, e segundo ele a contrologia fortalece o powerhouse, agindo nas musculaturas do tronco e beneficiando o corpo.

Segundo Junior AAP e Silva A (2020) relata que as intervenções de exercícios de Pilates conveniente pode melhorar significativamente a força muscular e flexibilidade do tronco em mulheres, onde foi observado que mulheres praticantes do método pilates apresentam força muscular do músculo transverso do abdômen com valores estatisticamente superiores quando comparadas ao grupo praticante de musculação. 
Resultados presentes no estudo de Martins TNO, et al. (2017), demonstrou que nas 22 voluntárias que a presença de quadro álgicos encontrados, teve um índice variaram de $22 \%$ a $55 \%$. Em relação as limitações funcionais foram vistas que quando o processo cirúrgico era realizado ipsilateral ao membro dominante os comprometimentos podem ser maiores. Nesse contexto, foi possível observar que a partir do aumento da amplitude de movimento e da força muscula podemos afirmar que a fisioterapia com o uso do método Pilates pode ter resultados positivos (GUGELMIN MRG, 2018).

No estudo de Dias M, et al. (2017) demonstra a relativa alta de afastamentos da atividade profissional, estando estes relacionados com o maior número de queixas físicas, o medo de se machucar, a diminuição de força nos MMSS, a menor agilidade, a indisposição para trabalhar, a sensação de braço pesado, a perda do movimento, a dor e, ainda, a elevada frequência de exames contribuíram para o ocorrido. Foi visto também comprometimentos na funcionalidade das pacientes, o que impossibilita a realização das atividades comum do cotidiano, gerando dificuldades movimentar o braço acima da altura do ombro, por exemplo, alcançar objetos ou pentear os cabelos.

Segundo o estudo de Fernandes ACS, et al. (2017) corrobora a importância das intervenções da fisioterapia ainda de forma precoce no pós-operatório, não só prevenir complicações como também reabilitar as pacientes mais cedo para atividades de vida diária, desempenhando um papel fundamental para as pacientes que foram submetidas a mastectomia.

Com base nas análises feitas por Rosa DF, et al. (2018) na atividade EMG dos músculos estudados, é possível concluir que na realização dos exercícios de flexo extensão do quadril realizados no solo (RollUp) e no Cadillac (RollBack), a maior de manda muscular foi exigida no exercício de solo para o músculo oblíquo externo nas duas fases do movimento, os efeitos da realização do método se dá pelo fato dos exercícios serem realizados a partir do emprego de cadeias musculares de forma simultânea, além do mais aumenta o nível de consciência corporal e motora, a utilização dos movimentos de forma eficiente, aumenta 0 desempenho muscular para que se consiga recuperar o movimento funcional.

O presente estudo corrobora com Sener HÖ, et al. (2017), que observou uma diferença estatisticamente significativa no grupo de Pilates direcionado a funcionalidade, acompanhado de aumento da independência funcional e redução no linfedema, em seu estudo com mulheres em tratamento de câncer de mama, distribuídas entre grupo controle, com realização de exercícios referentes ao linfedema e grupo Pilates. Espíndula RC, et al. (2017) apresentou dados estatísticos que compararam os exercícios do Pilates com os exercícios domiciliares e perceberam diferenças na funcionalidade com o uso do método em pacientes no tratamento do câncer de mama, assim para o autor os resultados encontrados eram previstos, pois, o Pilates é uma técnica que apresenta o profissional capacitado para orientar as pacientes na execução dos exercícios, como uma motivação a mais para elas e a participação de outras companheiras no estúdio de Pilates.

A execução dos exercícios do método foi demonstrada, nesse estudo, para as pacientes quanto à realização correta dos mesmos e seguida de um profissional, para garantir a realização de forma correta e eficiente visando os objetivos do estudo. Os exercícios do Pilates no estudo de Zengin AA, et al. (2016) considera ser eficaz na redução da dor, no aumento da amplitude de movimento e da força muscular resultando na independência funcional do membro superior afetado em sobreviventes do câncer de mama.

\section{CONCLUSÃO}

Foi constatado que houve melhora na funcionalidade, comprovando a eficácia do Método Pilates no tratamento do membro superior de mulheres submetidas à mastectomia Radical. Demostrando que após 24 sessões do programa de reabilitação proposto, baseado nos princípios e exercícios clássicos do método, houve melhora em todas as variáveis estudadas para esse grupo amostral. De acordo com o trabalho desenvolvido, pôde-se observar que são necessários mais estudos sobre o tema, visto que o número de casos de câncer de mama ainda é elevado e pelo tratamento cirúrgico interferir no aspecto físico das pacientes, produzindo sequelas físicas e emocionais. Assim, é sugerido a realização de pesquisas que correlacionem grupos de intervenção e controle, e com a utilização de um número amostral maior. 


\section{AGRADECIMENTOS}

Agradecemos a Clínica de Fisioterapia Especializada - Desportiva por permitir que esse projeto de pesquisa fosse realizado no local. Aos grupos Amigas do Peito e Laços e Lenços por nos ajudar e ceder as pacientes para a realização do projeto.

\section{REFERÊNCIAS}

1. ALMEIDA TG, et al. Experience of young women with breast cancer and mastectomized. Escola Anna Nery, 2015; 19(3): 432-438.

2. ALVES ERP, et al. Climatério: a intensidade dos sintomas e o desempenho sexual. Texto \& Contexto Enfermagem, 2015; 24(1): 64-71.

3. ASSIS CS, et al. Medida de independência funcional em pacientes com claudicação intermitente. Rev Esc Enferm USP. 2015; 5(49): 756-761.

4. AYRES M, et al. BioEstat 5.4: aplicações estatísticas nas áreas das ciências biológicas e médicas. Sociedade Civil Mamirauá, 2015.

5. Branco ANC, et al. Comparação da satisfação, motivação, flexibilidade edor muscular tardia entre método Pilates moderno emétodo Pilates instável, 2017; 24(4): 427-436.

6. CASASSOLA GM, et al. Intervenções fisioterapêuticas utilizadas na reabilitação funcional do membro superior de mulheres pós-mastectomia. Fisioterapia Brasil, 2020; 21(1): 93-103.

7. DIASA M, et al. Implicações das cirurgias de câncer de mama nas atividades profissionais. Cadernos Brasileiros de Terapia Ocupacional, 2017; 25(2): 325-332.

8. ESPÍNDULA RC, et al. Pilates for breast cancer: A systematic review and meta-analysis. Rev. Assoc. Med. Bras. 2017, 63(11): 1006-11.

9. FERNANDES ACS. Atuação da fisioterapia no pós-operatório da mastectomia na força muscular, linfedema e amplitude de movimento do ombro. Rev. Cient. de Ciências Apl. da FAIP. 2017; 4(7): 31-41.

10. FERREIRA TN, et al. O método pilates em pacientes com lombalgia. Saúde \& Ciência em ação. 2016; 2(1): 56-65.

11. FIREMAN KM, et al. Percepção das Mulheres sobre sua Funcionalidade e Qualidade de Vida após Mastectomia. Revista Brasileira de Cancerologia 2018; 64(4): 499-508.

12. GUERRA MR, et al. Sobrevida por câncer de mama e iniquidade em saúde. Rio de Janeiro: Cadernos de Saúde Pública. 2015; 31(8): 1673-84.

13. GUGELMIN MRG, et al. Recursos E Tratamentos Fisioterápicos Utilizados Em Linfedema Pós-Mastectomia Radical E Linfadenectomia: Revisão De Literatura. Arquivos Catarinenses de Medicina, 2018; 47(3): 174-182.

14. INSTITUTO NACIONAL DE CÂNCER (INCA). A situação do câncer de mama no Brasil: síntese de dados dos sistemas de informação, $2019 . \quad$ Disponível em: https://www.inca.gov.br/sites/ufu.sti.inca.local/files/media/document/a_situacao_ca_mama_brasil_2019.pdf. Acessado em: 09 de maio de 2020.

15. JESUS LT et al. Effects of the Pilates method on lung function, thoracoabdominal mobility and respiratory muscle strength: non-randomized placebo-controlled clinical trial. Fisioterapia e Pesquisa, 2015; 22(3): 213-222.

16. JUNIOR AAP E SILVA A. Comparação da força muscular e flexibilidade entre praticantes de Pilates e musculação. Revista Pesquisa em Fisioterapia, 2020; 10(3): 478-485.

17. LENA PR, et al. Perfil epidemiológico de mulheres mastectomizadas em umserviço de referência localizado no Vale do Taquari/RS. Revista de Epidemiologia e Controle de Infecção, 2019; (2): 122-128.

18. MINISTÉRIO DA SAÚDE. Diretrizes para a detecção precoce do câncer de mama no Brasil, 2015. Disponível em: http://www1.inca.gov.br/inca/Arquivos/livro_deteccao_precoce_final.pdf Acesso em: 10 de maio de 2017.

19. PEREIRA LKNV, et al. As principais abordagens fisioterapêuticas em pacientes mastectomizadas. Rev. Disc. Uniabeu. 2015; 3(6): p. 43-50.

20. ROSA EF, et al. Comparação da atividade eletromiográfica em músculos do powerhouse no roll up e roll back no solo e no Cadillac do método Pilates. Fisioterapia Brasil 2018;19(2):183-189 1

21. ŞENER HÖ et al. Effects of clinical Pilates exercises on patients developing lymphedema after breast cancer treatment: a randomized clinical trial. The journal of breast health, 2017; 13(1): 16-22.

22. ZENGIN AA, et al. Effectiveness of Pilates-based exercises on upper extremity disorders related with breast cancer treatment. European journal of cancer care, 2017; 26(6) 1-8. 